\title{
Axisymmetric natural frequencies of statically loaded annular plates
}

\author{
Eihab M. Abdel-Rahman, Waleed F. Faris and Ali H. Nayfeh \\ Department of Engineering Science and Mechanics, MC 0219, Virginia Polytechnic Institute and State University, \\ Blacksburg, VA 24061, USA \\ E-mail: anayfeh@vt.edu
}

Received 29 May 2002

Revised 12 May 2003

\begin{abstract}
We present a numerical procedure to solve the axisymmetric vibration problem of statically loaded annular plates. We use the von Kármán nonlinear plate model to account for large deformations and study the effect of static deflections on the natural frequencies and mode shapes for six combinations of boundary conditions. The shooting method is used to solve the resulting eigenvalue problem. Our results show that static deformations have a significant effect on the natural frequencies and small effect on the mode shapes of the plate. Further, the results show that the presence of in-plane stresses has a significant effect on the natural frequencies.
\end{abstract}

\section{Introduction}

Plate theories are classified as thin and thick plate theories. Thick plate theories, such as the Mindlin theory, account for shear stresses across the plate thickness and apply well to disc problems. Thin plate theories do not account for shear across the plate thickness. Thin plate theories are classified as linear and nonlinear theories. Linear theories account for small deflections only and do not account for in-plane stress effects. Nonlinear theories account for large deflections and in-plane effects. The most commonly used nonlinear thin plate theory is the von Kármán theory. Thin circular plates are used extensively in mechanical, civil, and nuclear engineering applications. Circular plates with a concentric hole or a rigid boss at the center are modeled as annular plates [1].

Assuming small deflections, Southwell [2] extended Kirchhoff's linear thin-plate theory to uniform annular plates. He derived the characteristic equation governing the transverse free vibrations of a clamped-free annular plate and solved it numerically. Hort and

\footnotetext{
*Corresponding author. E-mail: anayfeh@vt.edu.
}

Koenig [3] used this linear model to calculate the natural frequencies of annular plates subject to a few combinations of boundary conditions. Raju [4] used the same model to calculate the natural frequencies of an annular plate subject to the nine simple combinations of boundary conditions. Vogel and Skinner [5] carried out calculations for the same cases using a different numerical procedure. They also conducted experiments on free-clamped and clamped-free annular plates. The experimental results of the free-clamped plate matched those they obtained numerically, whereas the experimental results for the clamped-free plate did not match their numerical results.

There have seen several attempts in recent years to use various approximate methods to improve the accuracy of the results obtained with the linear models and compare the efficiency of these methods. Sonzogni et al. [6] used the Rayleigh-Ritz method to calculate the natural frequencies for an annular plate subject to four combinations of boundary conditions. Amabili et al. $[7,8]$ used the assumed mode method to calculate the natural frequencies for an annular plate subject to the nine simple combinations of boundary conditions. Gabrielson [9] compared the natural frequencies he obtained using the energy method to those obtained by 
evaluating the exact solution and found good agreement between both approaches. Wong et al. [10] used the mode subtraction method to calculate the natural frequencies and mode shapes of an annular plate subject to three combinations of boundary conditions. Liew and Yang [11] used the Raliegh-Ritz method, employing specially formulated trial functions derived from a three-dimensional elasticity model, to calculate the natural frequencies and mode shapes of an annular plate subject to the nine simple combinations of boundary conditions.

To the best of our knowledge, there has been no attempt to study the effect of large static deformations on the natural frequencies and modes shapes of annular plates. This information is of value to designers and structural analysts dealing with real-life structures that involve annular plates. In this paper, we have used the von Kármán thin-plate theory to account for large static deformations in axisymmetric annular plates. The natural frequencies and mode shapes are obtained numerically for a series of uniform loads from zero to a loading level where the maximum deflection is twice the plate thickness. We report the relations between the applied nondimensional loading, natural frequencies, deflection, and the associated changes in the mode shapes.

\section{Problem formulation}

\subsection{Governing equations}

We investigate the axisymmetric transverse natural frequencies of annular plates bending under a constant transverse load $p$. The axisymmetric equations of motion are

$$
\begin{aligned}
& D \nabla^{4} w+\rho h \frac{\partial^{2} w}{\partial t^{2}}= \frac{1}{r}\left(\frac{\partial^{2} w}{\partial r^{2}} \frac{\partial \Phi}{\partial r}+\frac{\partial w}{\partial r} \frac{\partial^{2} \Phi}{\partial r^{2}}\right) \\
&+p \\
& \nabla^{4} \Phi=-\frac{E h}{r} \frac{\partial^{2} w}{\partial r^{2}} \frac{\partial w}{\partial r}
\end{aligned}
$$

where $w(r, t)$ is the transverse deflection, $t$ is time, $\rho$ is the material density, $h$ is the plate thickness, and $\Phi$ is the stress function. The modulus of rigidity $D$ is

$$
D=\frac{E h^{3}}{12\left(1-\nu^{2}\right)}
$$

where $E$ is Young's modulus and $\nu$ is Poisson's ratio. The axisymmetric differential operator $\nabla^{4}$ is

$$
\nabla^{4}=\frac{\partial^{4}}{\partial r^{4}}+\frac{2}{r} \frac{\partial^{3}}{\partial r^{3}}-\frac{1}{r^{2}} \frac{\partial^{2}}{\partial r^{2}}+\frac{1}{r^{3}} \frac{\partial}{\partial r}
$$

We introduce nondimensional variables, denoted by hats, and defined as follows:

$$
\begin{aligned}
& \hat{r}=\frac{r}{R}, \hat{w}=\frac{w}{h}, \hat{\Phi}=\frac{\Phi}{E h^{3}}, \\
& \hat{p}=12\left(1-\nu^{2}\right) \frac{p}{E}, \hat{t}=\sqrt{\frac{D}{\rho h}} \frac{t}{R^{2}}
\end{aligned}
$$

where $R$ is the outer radius of the annular plate. Substituting Eq. (4) into Eqs (1) and (2) and dropping the hats, we obtain

$$
\begin{aligned}
& \nabla^{4} w+\frac{\partial^{2} w}{\partial t^{2}}=\frac{12\left(1-\nu^{2}\right)}{r} \\
& \left(\frac{\partial^{2} w}{\partial r^{2}} \frac{\partial \Phi}{\partial r}+\frac{\partial w}{\partial r} \frac{\partial^{2} \Phi}{\partial r^{2}}\right)+\alpha p \\
& \nabla^{4} \Phi=-\frac{1}{2 r} \frac{\partial}{\partial r}\left(\frac{\partial w}{\partial r}\right)^{2}
\end{aligned}
$$

where

$$
\alpha=\left(\frac{R}{h}\right)^{4}
$$

We express the plate deflection and the stress function as the summation of static components, denoted by $w_{s}(r)$ and $\Phi_{s}(r)$, and dynamic components, denoted by $u(r, t)$ and $\phi(r, t)$; that is,

$$
\begin{aligned}
& w(r, t)=w_{s}(r)+u(r, t) \\
& \Phi(r, t)=\Phi_{s}(r)+\phi(r, t)
\end{aligned}
$$

To calculate the static deflection, we set the time derivative in Eq. (5) equal to zero and obtain

$$
\begin{aligned}
\nabla^{4} w_{s}= & \frac{12\left(1-\nu^{2}\right)}{r} \\
& \left(\frac{\partial^{2} w_{s}}{\partial r^{2}} \frac{\partial \Phi_{s}}{\partial r}+\frac{\partial w_{s}}{\partial r} \frac{\partial^{2} \Phi_{s}}{\partial r^{2}}\right)+\alpha p \\
\nabla^{4} \Phi_{s}= & -\frac{1}{2 r} \frac{\partial}{\partial r}\left(\frac{\partial w_{s}}{\partial r}\right)^{2}
\end{aligned}
$$

We solve the boundary-value problem composed of these two equations and the corresponding boundary conditions for $w_{s}(r)$ and $\Phi_{s}(r)$.

We generate the equations of motion governing the dynamic behavior of the plate around the deflected shape by substituting Eqs (7) and (8) into Eqs (5) and (6) and dropping the terms representing the equilibrium position. The result is 


$$
\begin{aligned}
\nabla^{4} u+\frac{\partial^{2} u}{\partial t^{2}}= & \frac{12\left(1-\nu^{2}\right)}{r} \\
& \left(\frac{\partial^{2} u}{\partial r^{2}} \frac{\partial \Phi_{s}}{\partial r}+\frac{\partial^{2} w_{s}}{\partial r^{2}} \frac{\partial \phi}{\partial r}\right. \\
& +\frac{\partial u}{\partial r} \frac{\partial^{2} \Phi_{s}}{\partial r^{2}}+\frac{\partial w_{s}}{\partial r} \frac{\partial^{2} \phi}{\partial r^{2}} \\
& \left.+\frac{\partial^{2} u}{\partial r^{2}} \frac{\partial \phi}{\partial r}+\frac{\partial u}{\partial r} \frac{\partial^{2} \phi}{\partial r^{2}}\right) \\
\nabla^{4} \phi=-\frac{1}{2 r} \frac{\partial}{\partial r} & {\left[2 \frac{\partial u}{\partial r} \frac{\partial w_{s}}{\partial r}+\left(\frac{\partial u}{\partial r}\right)^{2}\right] }
\end{aligned}
$$

To calculate the natural frequencies and corresponding mode shapes, we solve the linearized eigenvalue problem. To do this, we drop the nonlinear terms in Eqs (11) and (12), and obtain

$$
\begin{aligned}
\nabla^{4} u+\frac{\partial^{2} u}{\partial t^{2}}= & \frac{12\left(1-\nu^{2}\right)}{r} \\
& \left(\frac{\partial^{2} u}{\partial r^{2}} \frac{\partial \Phi_{s}}{\partial r}+\frac{\partial^{2} w_{s}}{\partial r^{2}} \frac{\partial \phi}{\partial r}\right. \\
& \left.+\frac{\partial u}{\partial r} \frac{\partial^{2} \Phi_{s}}{\partial r^{2}}+\frac{\partial w_{s}}{\partial r} \frac{\partial^{2} \phi}{\partial r^{2}}\right) \\
\nabla^{4} \phi=-\frac{1}{r} \frac{\partial}{\partial r} & \left(\frac{\partial u}{\partial r} \frac{\partial w_{s}}{\partial r}\right)
\end{aligned}
$$

To solve Eqs (13) and (14) subject to the proper boundary conditions for the undamped natural frequencies and mode shapes of the plate, we assume a harmonic motion in the $n t h$ mode in the form

$$
\begin{aligned}
& u(r, t)=\psi_{n}(r) e^{i \omega_{n} t} \\
& \phi(r, t)=\chi_{n}(r) e^{i \omega_{n} t}
\end{aligned}
$$

where $\psi_{n}(r)$ is the $n t h$ mode shape, $\chi_{n}(r)$ is the associated stress function, and $\omega_{n}$ is the $n t h$ nondimensional natural frequency. Substituting Eqs (15) and (16) into Eqs (13) and (14) yields

$$
\begin{aligned}
\nabla^{4} \psi_{n}= & \frac{12\left(1-\nu^{2}\right)}{r}\left(\psi_{n}^{\prime \prime} \Phi_{s}^{\prime}+w_{s}^{\prime \prime} \chi_{n}^{\prime}\right. \\
& \left.+\psi_{n}^{\prime} \Phi_{s}^{\prime \prime}+w_{s}^{\prime} \chi_{n}^{\prime \prime}\right)+\omega^{2} \psi_{n} \\
\nabla^{4} \chi_{n}= & -\frac{1}{r} \frac{\partial}{\partial r}\left(w_{s}^{\prime} \psi_{n}^{\prime}\right)
\end{aligned}
$$

Substituting Eqs (15) and (16) into the boundary conditions produces the boundary conditions relevant to the eigenvalue problem. To solve this problem, we release one of the geometric boundary conditions and solve the boundary-value problem iteratively using a shooting method until $\omega_{n}, \psi_{n}$, and $\chi_{n}$ converge to within predefined tolerances. This procedure is repeated for each mode shape and associated natural frequency and stress function, using various sets of initial guesses for the mode shape, natural frequency, and stress function.

\subsection{Boundary conditions}

There are two types of boundary conditions applicable to the nonlinear plate problem: those associated with the transverse deflection and those associated with the in-plane forces. The first type of boundary conditions is

For a clamped edge

$$
w=0 \text { and } \frac{\partial w}{\partial r}=0
$$

For a simply-supported edge

$$
w=0 \text { and } \frac{\partial^{2} w}{\partial r^{2}}+\frac{\nu}{r} \frac{\partial w}{\partial r}=0
$$

For a free edge

$$
\begin{aligned}
& \frac{\partial^{2} w}{\partial r^{2}}+\frac{\nu}{r} \frac{\partial w}{\partial r}=0 \\
& \frac{\partial^{3} w}{\partial r^{3}}+\frac{1}{r} \frac{\partial^{2} w}{\partial r^{2}}-\frac{1}{r^{2}} \frac{\partial w}{\partial r}=0
\end{aligned}
$$

The second type of boundary conditions is For a movable edge

$$
\frac{\partial \Phi}{\partial r}=0
$$

For an immovable edge

$$
\begin{aligned}
& \frac{\partial^{2} \Phi}{\partial r^{2}}-\frac{\nu}{r} \frac{\partial \Phi}{\partial r}=0 \\
& \frac{\partial^{3} \Phi}{\partial r^{3}}+\frac{1}{r} \frac{\partial^{2} \Phi}{\partial r^{2}}-\frac{1}{r^{2}} \frac{\partial \Phi}{\partial r}=0
\end{aligned}
$$

Whereas a free edge is movable, a clamped edge is immovable, and a simply-supported edge can be either movable (sliding) or immovable (hinged).

\section{Results}

Results were obtained for annular plates in each of the following boundary conditions 


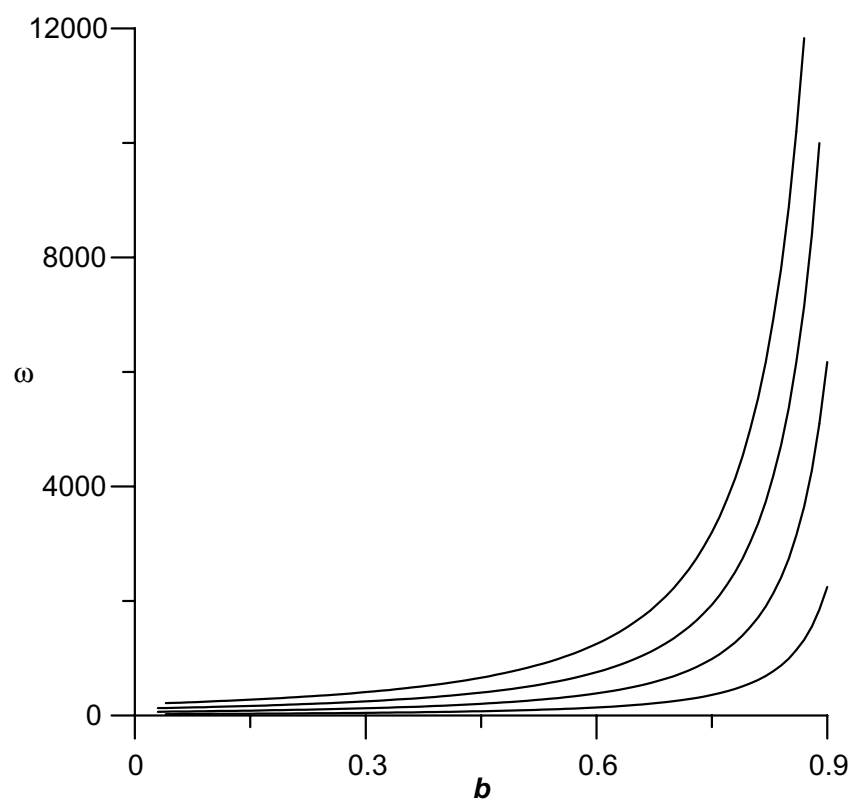

Fig. 1. Variation of the first four natural frequencies with the inner radius for a flat clamped-clamped plate.

\section{Clamped-clamped plate}

The plate is clamped on both edges. The boundary conditions at $r=b$ and 1 are described by Eqs (19) and (23). To verify our procedure, we generated the first four natural frequencies of a flat clamped-clamped plate of varying inner radii, Fig. 1. Comparison of these results to the first two axisymmetric natural frequencies reported by Leissa [12] shows good agreement.

Figure 2 shows variation of the maximum nondimensional deflection $W_{\text {Max }}$ with the applied nondimensional load $\alpha p$ for inner radii of $b=0.1,0.3,0.5$, and 0.7 . Figure 3 shows the first (a) and second (b) natural frequencies $\omega_{1}$ and $\omega_{2}$ of the plate as functions of the maximum nondimensional deflection for inner radii of $b=0.1,0.3,0.5$, and 0.7. Comparison of the two figures show that increasing the deflection increases both natural frequencies, however this effect is more pronounced in the first natural frequency than it is in the second natural frequency.

\section{Free-clamped plate}

The plate is free on the outer edge and clamped on the inner edge. The boundary conditions at the free edge $r=1$ are described by Eqs (21) and (22), while the boundary conditions at the clamped edge $r=b$ are described by Eqs (19) and (23). Figure 4 shows variation of the maximum nondimensional deflection with the applied nondimensional load. Figure 5 shows the first and second natural frequencies of the plate as functions of the maximum nondimensional deflection.

\section{Clamped-free plate}

The plate is clamped on the outer edge and free on the inner edge. The boundary conditions at the clamped edge $r=1$ are described by Eqs (19) and (23), while the boundary conditions at the free edge $r=b$ are described by Eqs (21) and (22). Figure 6 shows variation of the maximum nondimensional deflection with the applied nondimensional load. Figure 7 shows the first and second natural frequencies of the plate as functions of the maximum nondimensional deflection. We note that clamping the outer edge of the plate stiffens the plate response more than clamping the inner edge of plate. Comparison of the results in this case with those in the previous case show, as expected, a smaller deflection for the same forcing level and a higher natural frequency for the same deflection level.

\section{Simply supported-simply supported plate}

The plate is simply-supported on both edges where the outer edge is hinged and the inner edge is sliding. The boundary conditions at the hinged edge $r=1$ are described by Eqs (20) and (23), while the boundary conditions at the sliding edge $r=b$ are described by Eqs (20) and (22). Figure 8 shows variation of the maximum nondimensional deflection with the applied nondimensional load. Figure 9 shows the first and second natural frequencies of the plate as functions of the maximum nondimensional deflection. 


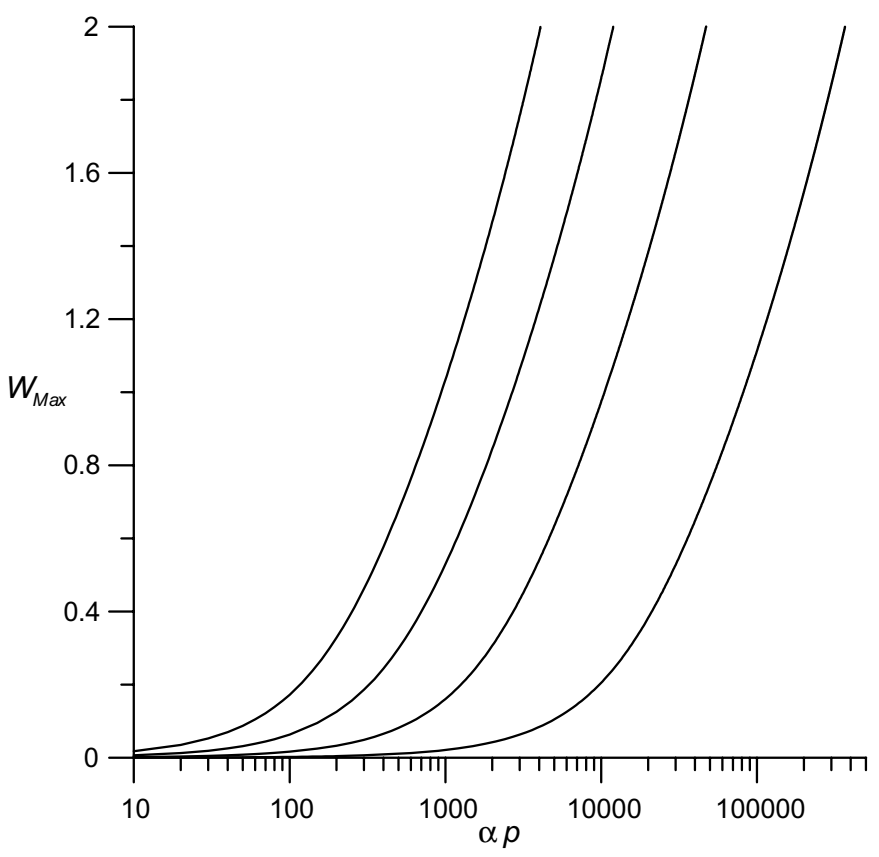

Fig. 2. Variation of the maximum nondimensional deflection $W_{\mathrm{Max}}$ with the applied nondimensional load $\alpha p$ for clamped-clamped plates of inner radii $b=0.1,0.3,0.5$, and 0.7 .

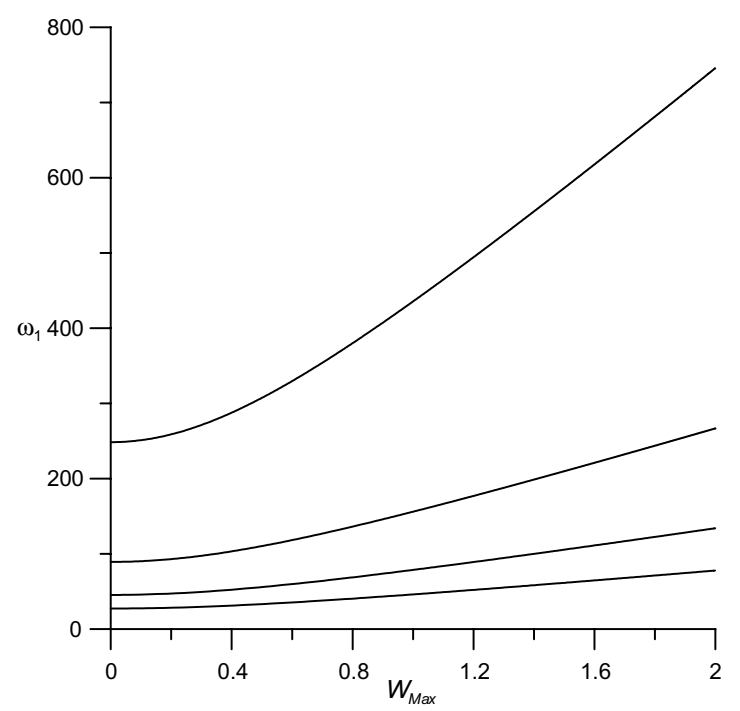

(a)

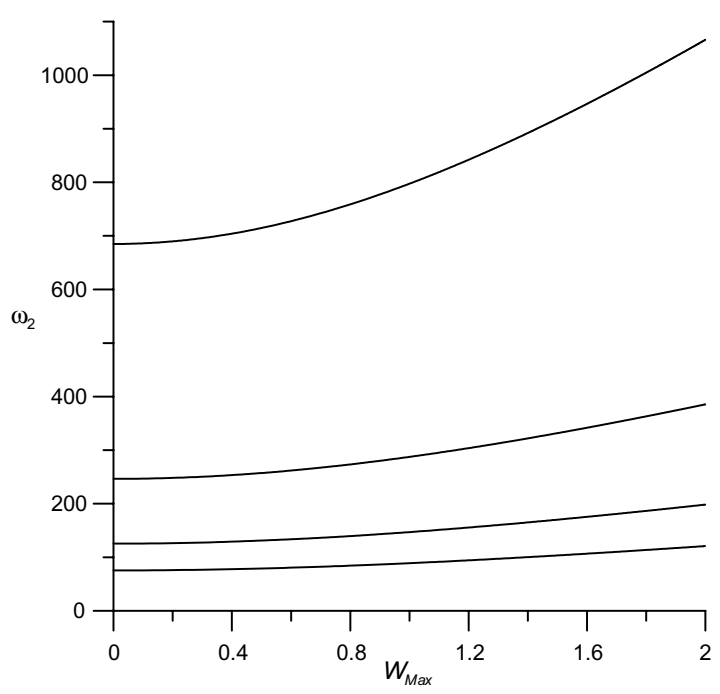

(b)

Fig. 3. The first (a) and second (b) natural frequencies of the plate as functions of the maximum nondimensional deflection $W_{\text {Max }}$ for clamped-clamped plates of inner radii $b=0.1,0.3,0.5$, and 0.7 .

\section{Simply supported (sliding)-clamped plate}

The plate has a sliding simple support on the outer edge and is clamped on the inner edge. The boundary conditions at the sliding edge $r=1$ are described by Eqs (20) and (22), while the boundary conditions at the clamped edge $r=b$ are described by Eqs (19) and (23). Figure 10 shows variation of the maximum nondimensional deflection with the applied nondimensional load. Figure 11 shows the first and second natural frequencies of the plate as functions of the maximum nondi- 


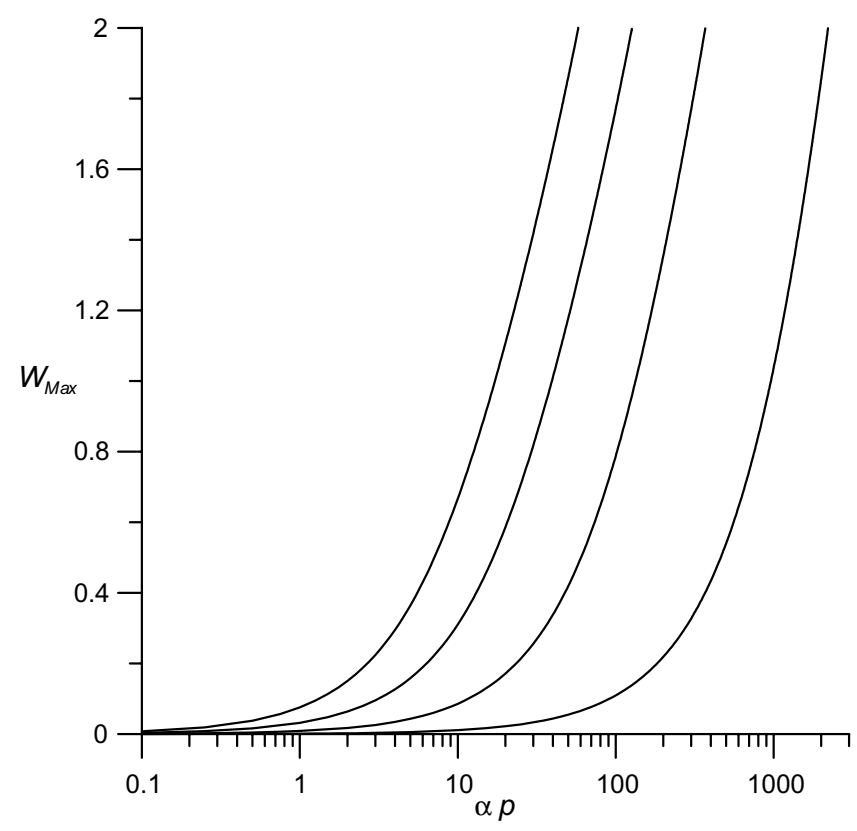

Fig. 4. Variation of the maximum nondimensional deflection $W_{\mathrm{Max}}$ with the applied nondimensional load $\alpha p$ for free-clamped plates of inner radii $b=0.1,0.3,0.5$, and 0.7 .

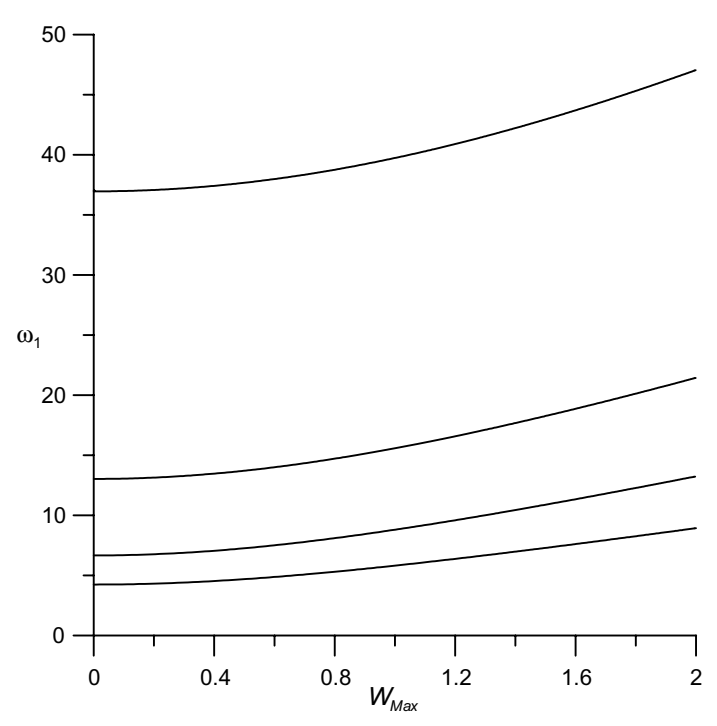

(a)

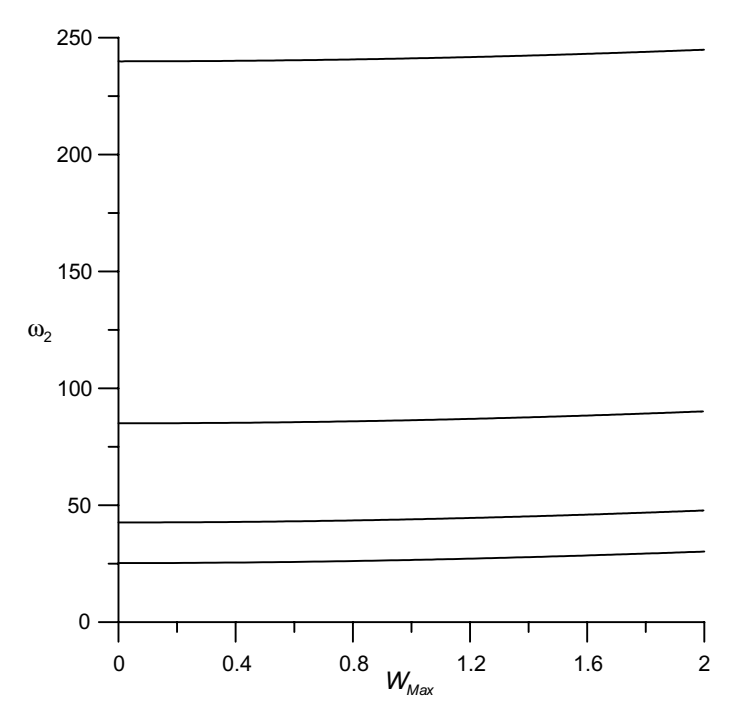

(b)

Fig. 5. The first (a) and second (b) natural frequencies of the plate as functions of the maximum nondimensional deflection $W_{\mathrm{M}}$ ax for free-clamped plates of inner radii $b=0.1,0.3,0.5$, and 0.7 .

mensional deflection.

\section{Simply supported (hinged)-clamped plate}

The plate has a hinged simple support on the outer edge and is clamped on the inner edge. The boundary conditions at the hinged edge $r=1$ are described by Eqs (20) and (23), while the boundary conditions at the clamped edge $r=b$ are described by Eqs (19) and (23). Figure 12 shows variation of the maximum nondimensional deflection with the applied nondimensional load. Figure 13 shows the first and second natural frequencies of the plate as functions of the max- 


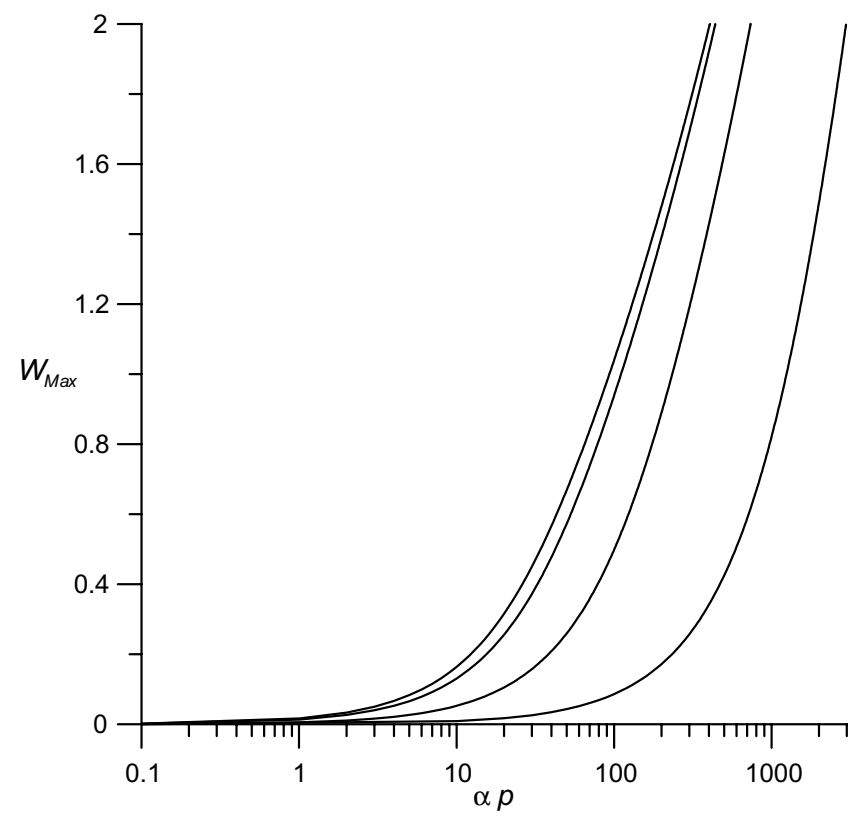

Fig. 6. Variation of the maximum nondimensional deflection $W_{\mathrm{Max}}$ with the applied nondimensional load $\alpha p$ for clamped-free plates of inner radii $b=0.1,0.3,0.5$, and 0.7 .

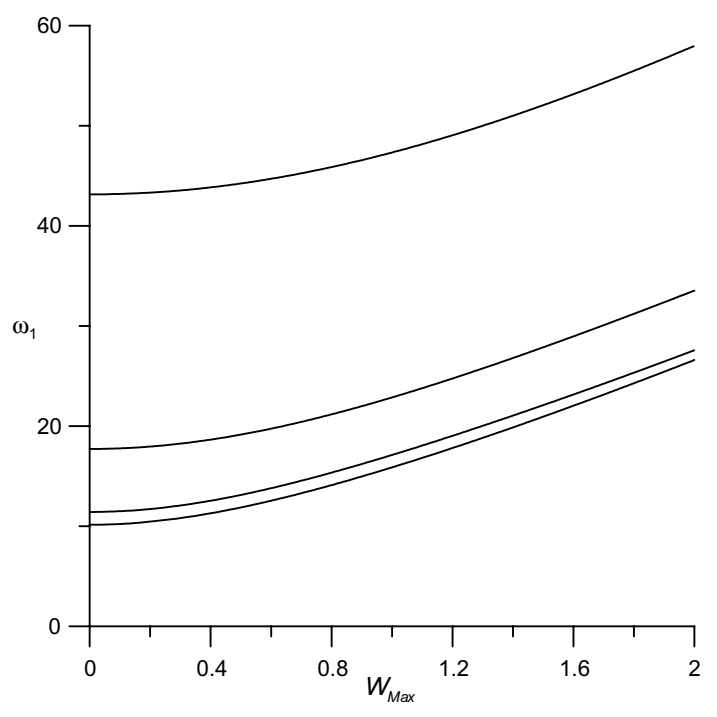

(a)

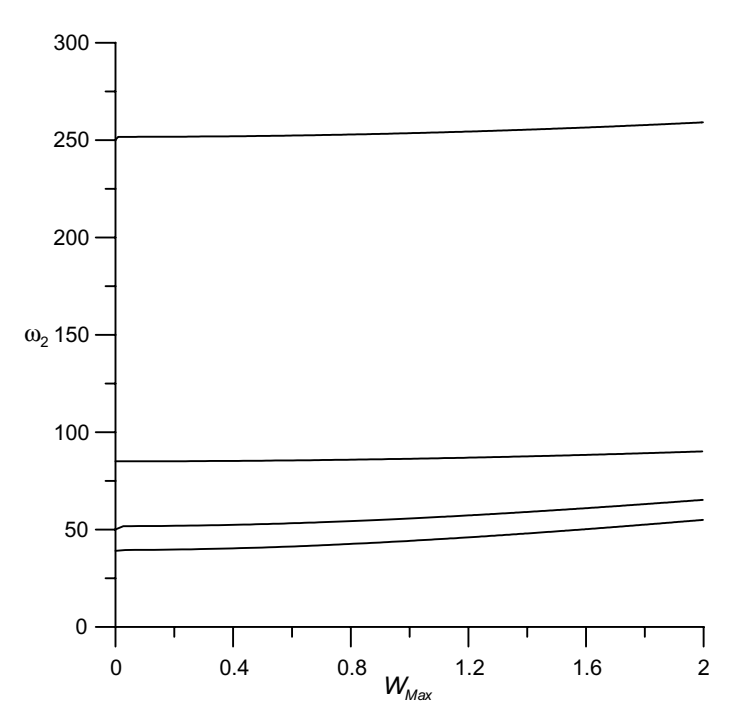

(b)

Fig. 7. The first (a) and second (b) natural frequencies of the plate as functions of the maximum nondimensional deflection $W_{\mathrm{Max}}$ for clamped-free plates of inner radii $b=0.1,0.3,0.5$, and 0.7 .

imum nondimensional deflection. Comparison of the results of this case to those of the previous case show significant differences in both the deflection and natural frequency. For a flat plate, the hinged boundary condition produces higher natural frequencies than the corresponding sliding boundary condition for all of the inner radii cases presented. This is expected because the hinged (immovable) boundary condition is stiffer than the sliding (movable) boundary condition. As the plate deflection increases, the natural frequencies corresponding to a sliding boundary condition increase more rapidly than those corresponding to a hinged boundary 


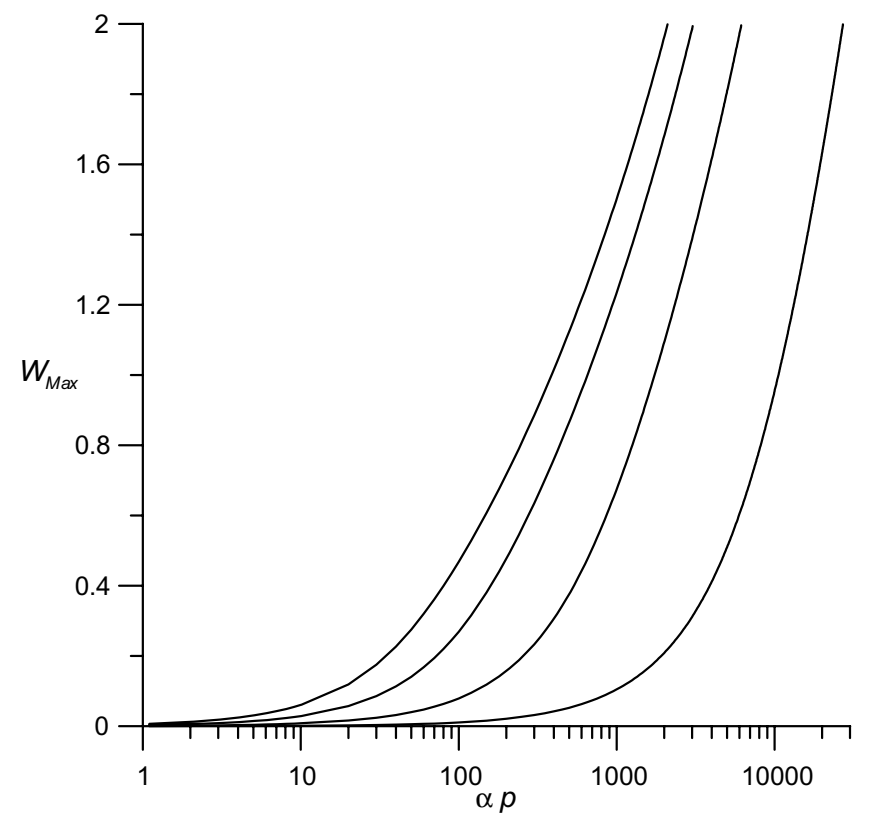

Fig. 8. Variation of the maximum nondimensional deflection $W_{\mathrm{Max}}$ with the applied nondimensional load $\alpha p$ for simply supported-simply supported plates of inner radii $b=0.1,0.3,0.5$, and 0.7 .

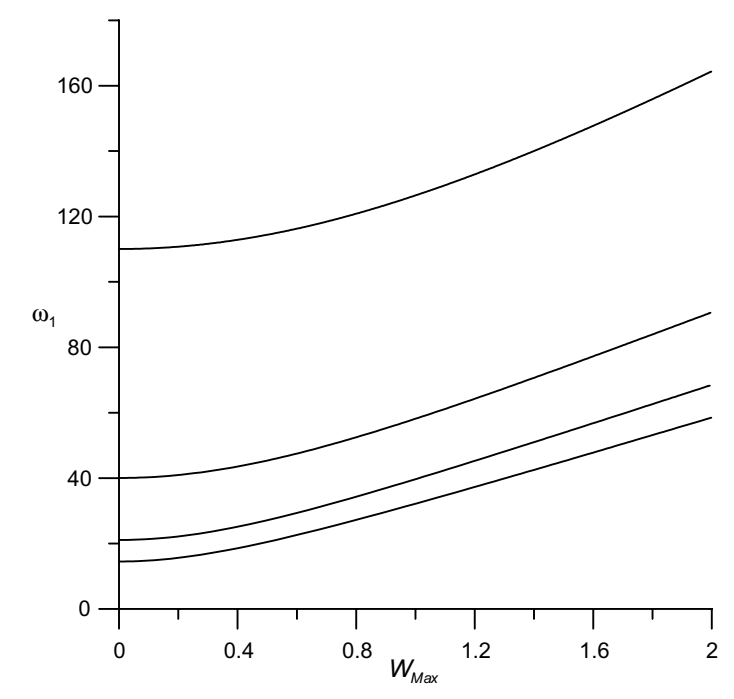

(a)

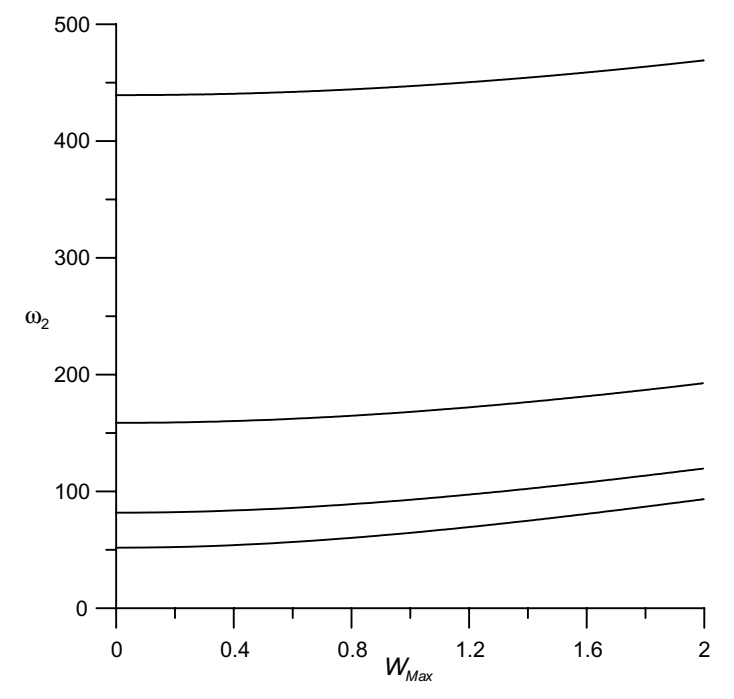

(b)

Fig. 9. The first (a) and second (b) natural frequencies of the plate as functions of the maximum nondimensional deflection Wax for simply supported-simply supported plates of inner radii $b=0.1,0.3,0.5$, and 0.7 .

condition. At a deflection level of $W_{\mathrm{Max}}=2$, the natural frequencies corresponding to the sliding boundary condition are significantly higher, up to $363 \%$, than those corresponding to the hinged boundary condition. This is also expected since attaining the same level of plate deflection under the sliding boundary condition requires higher applied loads, and thus generating more in-plane forces, than those for a hinged boundary condition. The in-plane forces, in turn, increase the stiffness and raise the natural frequencies of the plate. These results indicate the critical importance of including the in-plane forces in the plate model and correctly identifying simply-supported boundary conditions as either sliding or hinged. 


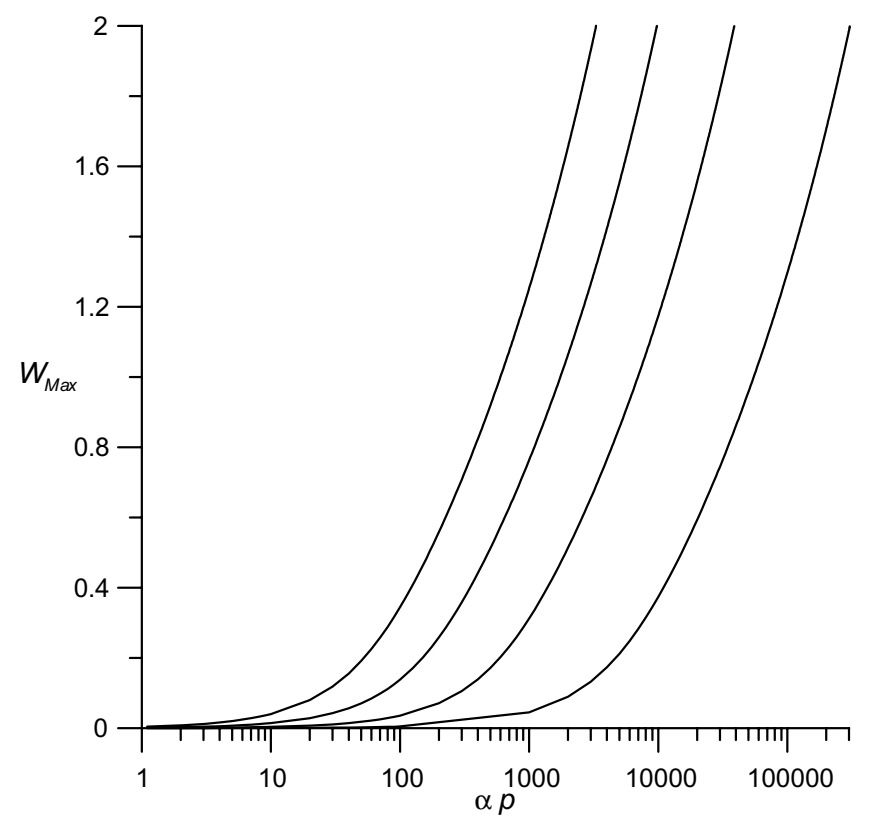

Fig. 10. Variation of the maximum nondimensional deflection $W_{\text {Max }}$ with the applied nondimensional load $\alpha p$ for sliding-clamped plates of inner radii $b=0.1,0.3,0.5$, and 0.7 .

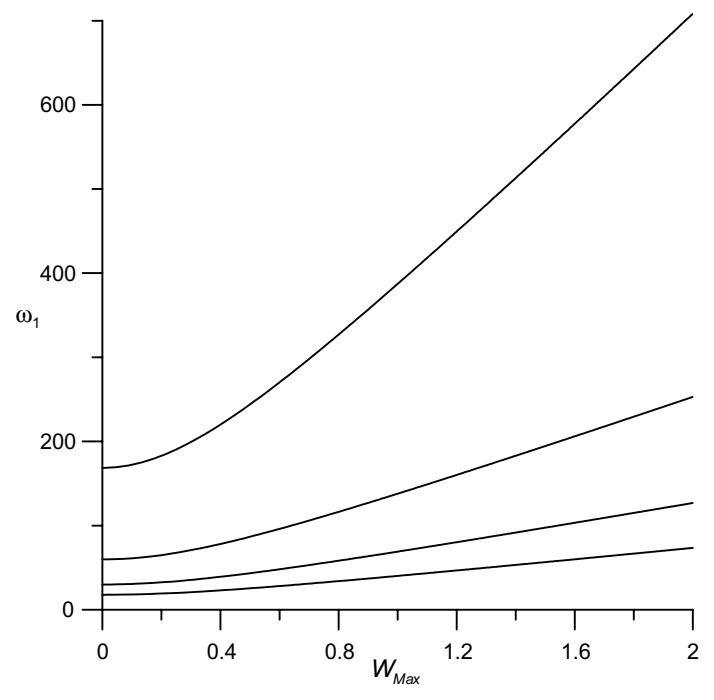

(a)

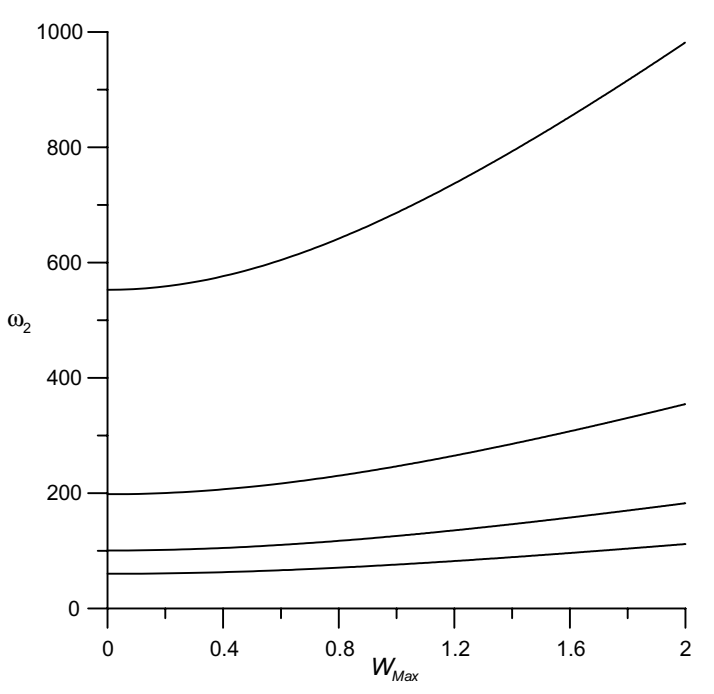

(b)

Fig. 11. The first (a) and second (b) natural frequencies of the plate as functions of the maximum nondimensional deflection $W_{\mathrm{M}}$ ax for sliding-clamped plates of inner radii $b=0.1,0.3,0.5$, and 0.7 .

Figures 2, 4, 6, 8, 10 and 12 show that the relationship between the load and deflection starts strongly nonlinear. This nonlinearity becomes less pronounced as the load increases. Examination of Eq. (9) helps to explain these results. As the forcing term in the equation increases, it dominates the nonlinear terms in the equations.
Figures 3, 5, 7, 9, 11 and 13 show that the natural frequencies increase as the inner radius (and thus the stiffness of the plate) increases for the same value of deflection. The results also show a significant increase in the natural frequency with deflection. The rate of increase of the natural frequencies depends on the nature of the plate boundary conditions. Comparison of 


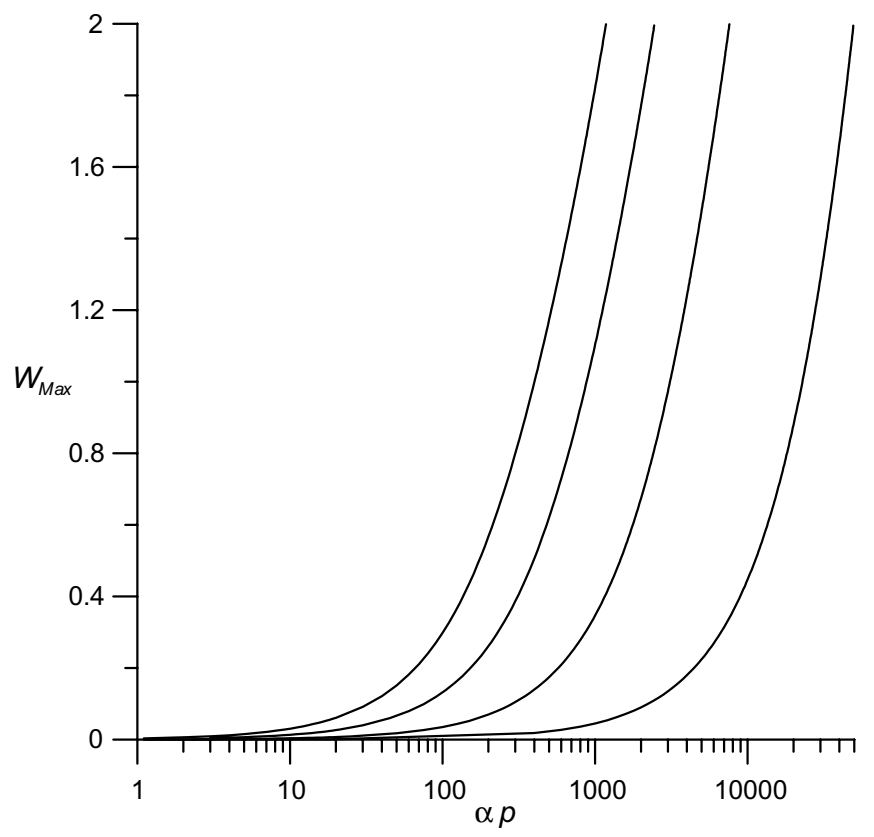

Fig. 12. Variation of the maximum nondimensional deflection $W_{\text {Max }}$ with the applied nondimensional load $\alpha p$ for hinged-clamped plates of inner radii $b=0.1,0.3,0.5$, and 0.7 .

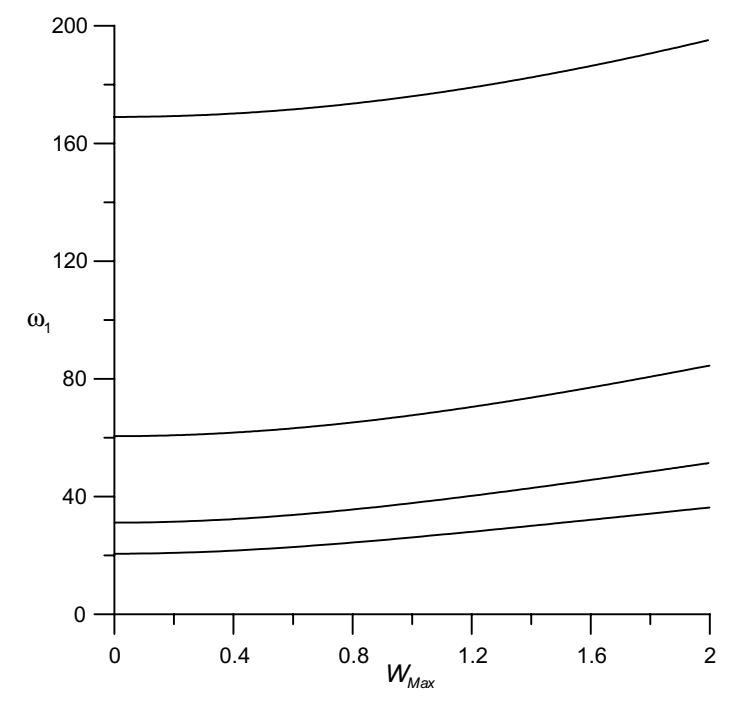

(a)

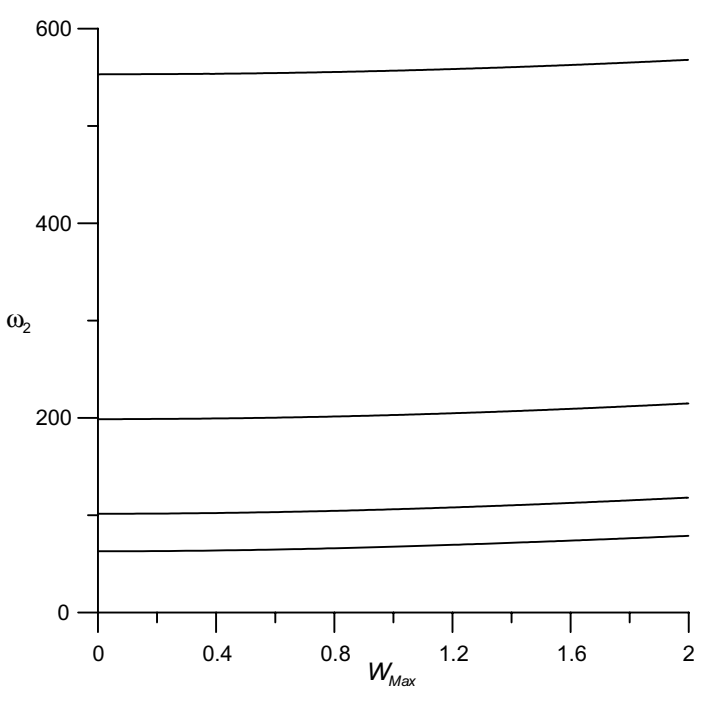

(b)

Fig. 13. The first (a) and second (b) natural frequencies of the plate as functions of the maximum nondimensional deflection Waax for hinged-clamped plates of inner radii $b=0.1,0.3,0.5$, and 0.7 .

the changes in the first natural frequency to those in the second natural frequency show that the first natural frequency is more sensitive to deflection than the second.

While the linear plate theory is traditionally used in the literature for deflections smaller than plate thick- ness, i.e. the natural frequency is assumed constant within these limits, our results show differences, in the sliding-clamped plate case, of up to $231 \%$ between $\omega_{1}$ for a flat plate and that for a plate undergoing a maximum deflection equal to its thickness. Further, a consistently nonlinear relationship between the natural 


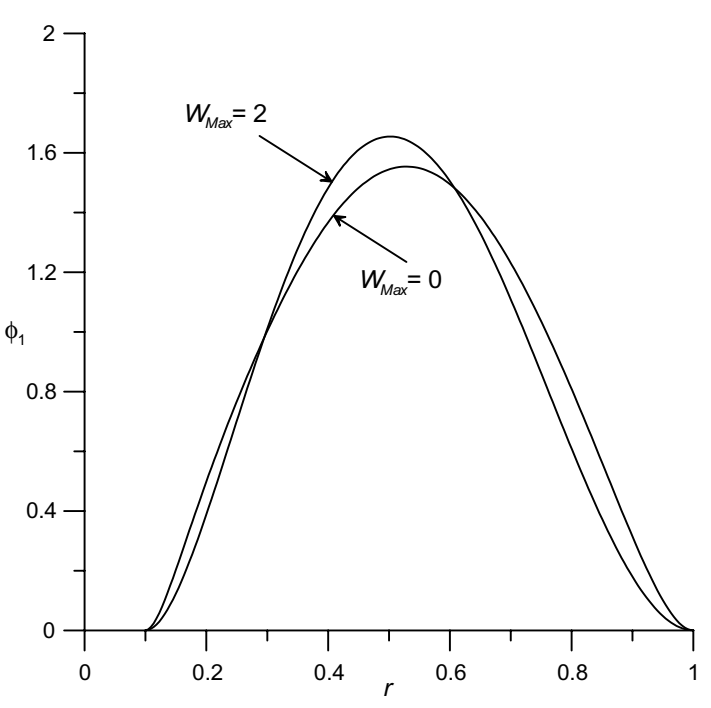

(a)

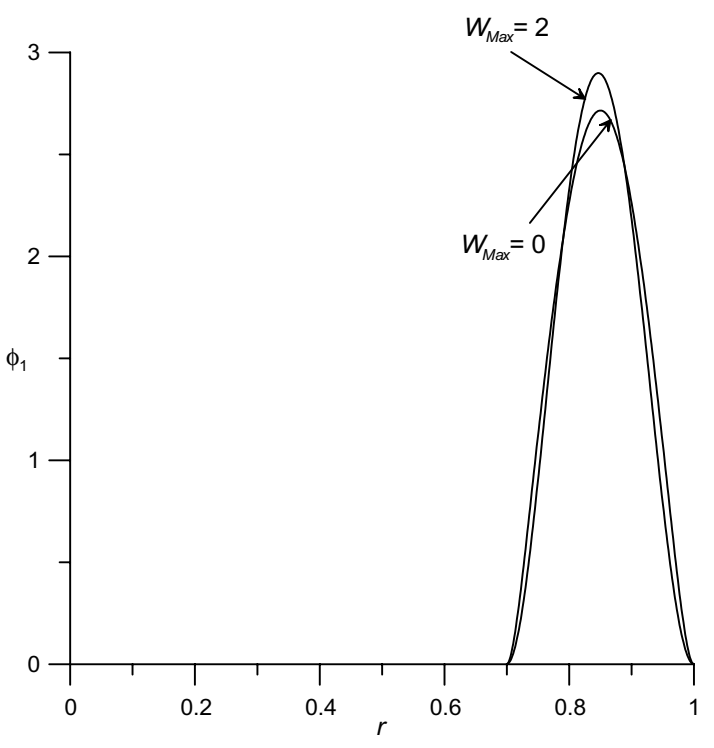

(b)

Fig. 14. The first mode shape for flat and deflected $\left(W_{\mathrm{Max}}=2\right)$ clamped-clamped plate of inner radius (a) $b=0.1$ and $(\mathrm{b}) b=0.7$.

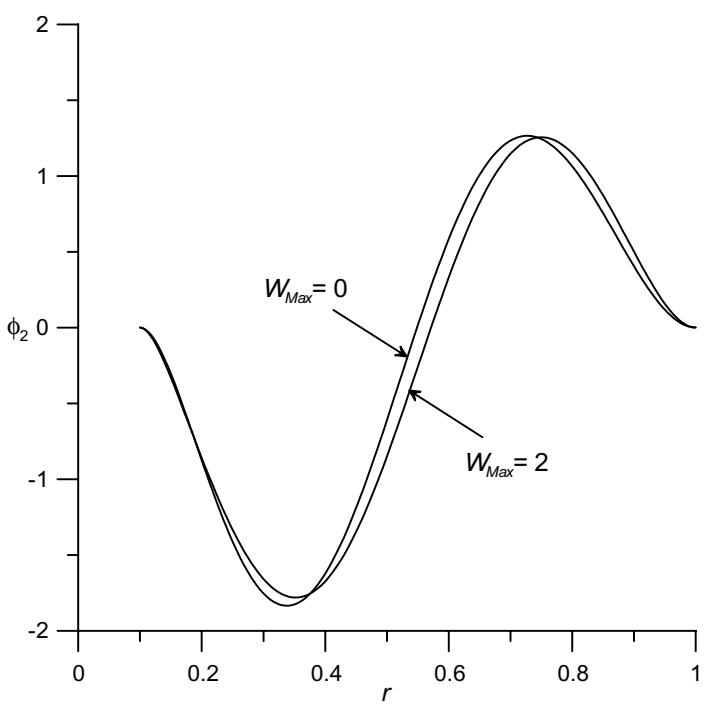

(a)

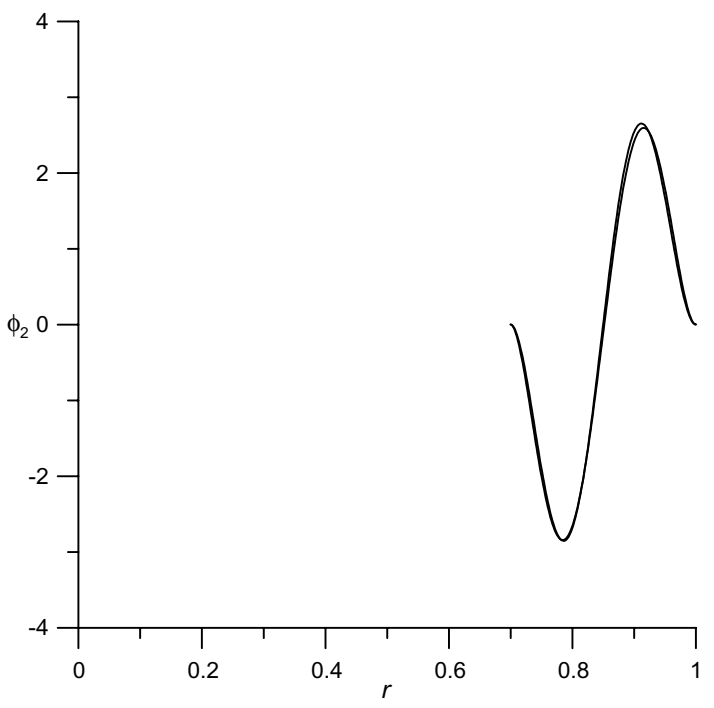

(b)

Fig. 15. The second mode shape for flat and deflected $\left(W_{\mathrm{Max}}=2\right)$ clamped-clamped plate of inner radius (a) $b=0.1$ and (b) $b=0.7$.

frequency and the deflection is observed across all the loading cases. The nonlinearity starts strong and becomes less pronounced with further deflection. These changes in the values of the natural frequencies raise the possibility of internal resonances, not present in the flat plate, developing as a result of deflection.

We obtained the mode shapes for the cases described above as the plate deflects from a straight position to a position where $W_{\mathrm{Max}}=2$. It was found that while quantitative changes were observed with plate deflection regardless of how stiff are the boundary conditions, no qualitative changes were observed in the mode shapes. Figures 14 and 15 show the first and second mode shapes, respectively, for the stiffest plate set-up (clamped-clamped boundary conditions) for a flat and a deflected $\left(W_{\mathrm{Max}}=2\right)$ plate in each case. 


\section{Conclusions}

We presented a numerical procedure to solve the axisymmetric vibrations problem of annular plates. The model accounts for the geometric nonlinearities due to large deformations. We used this procedure to obtain the natural frequencies and linear undamped mode shapes for six combinations of boundary conditions. Comparison of the first two natural frequencies of a clamped-clamped flat plate to those available in the literature shows good agreement, thus verifying our procedure. Our results show that large static deflections have a significant effect on the natural frequencies of the plate. In contrast to the predictions of the linear theory, the natural frequencies were found to increase significantly with deflections less than the thickness of the plate, thus putting the use of the linear theory, in that range, in question. On the other hand, small changes were found in the linear undamped mode shapes with deflection. In conclusion, the results show that the in-plane forces contribute significantly to the system behavior.

\section{References}

[1] H.Y. Su, K.S. Chen, D.C. Roberts and S.M. Spearing, Large deflection analysis of pre-stressed annular plates with rigid boss under axisymmetric loading, Journal of Micromechanics and Microengineering 11 (2001), 645-653.

[2] R.V. Southwell, On the free transverse vibrations of a uniform circular disk clamped at its center; and on the effects of rotation, Proceedings of the Royal Society 101 (1922), 133-153.

[3] W. Hort and M. Koenig, Studien über schwingungen von kreisplatten und ringen, Zeitschrift für Technische Physik 10 (1928), 373-382.

[4] N.P. Raju, Vibrations of annular plates, Journal of the Aeronautical Society of India 14 (1962), 37-52.

[5] S.M. Vogel and D.W. Skinner, Natural frequencies of transversely vibrating uniform annular plates, Journal of Applied Mechanics 32 (1965), 926-931.

[6] V. Sonzogni, R.H. Guiterrez and P.A.A. Laura, A note on numerical experiments in transverse vibrations of annular circular plates, Journal of Sound and Vibration 191 (1996), 167170.

[7] M. Amabili, G. Frosali and M.K. Kwak, Free vibrations of annular plates coupled with fluids, Journal of Sound and Vibration 191 (1996), 825-846.

[8] M. Amabili and G. Dalpiaz, Vibrations of base plates in annular cylindrical tanks: Theory and experiments, Journal of Sound and Vibration 211 (1998), 329-350.

[9] T.B. Gabrielson, Frequency constants for transverse vibration of annular disks, Journal of Acoustical Society of America 105 (1999), 3311-3317.

[10] W.O. Wong, L.H. Yam, Y.Y. Li, L.Y. Law and K.T. Chan, Vibration analysis of annular plates using mode subtraction method, Journal of Sound and Vibration 232 (2000), 807-822.

[11] K.M. Liew and B. Yang, Elasticity solutions for free vibrations of annular plates from three-dimensional analysis, International Journal of Solids and Structures 37 (2000), 7689-7702.

[12] A. Leissa, Vibration of Plates, American Institute of Physics, 1993. 

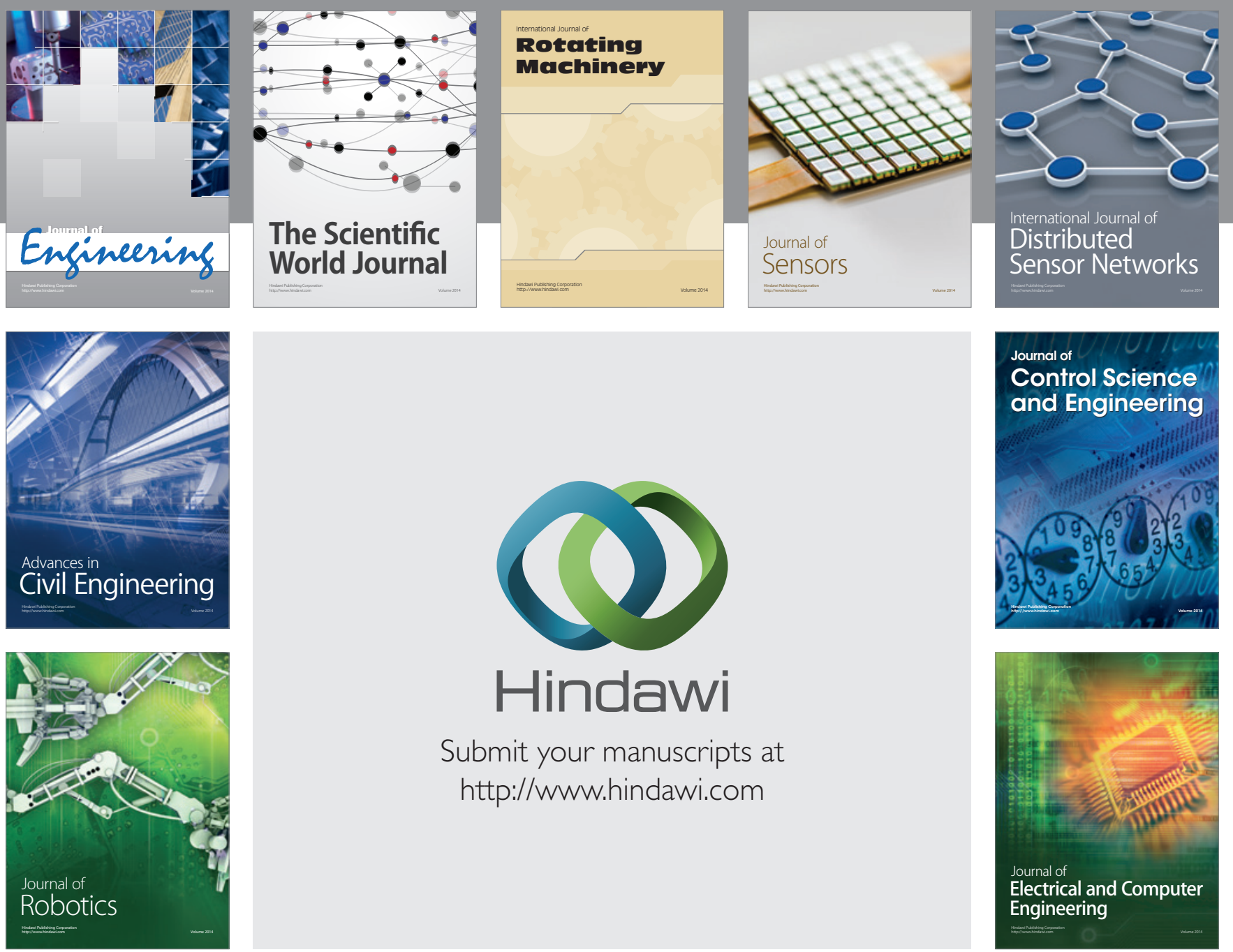

Submit your manuscripts at

http://www.hindawi.com
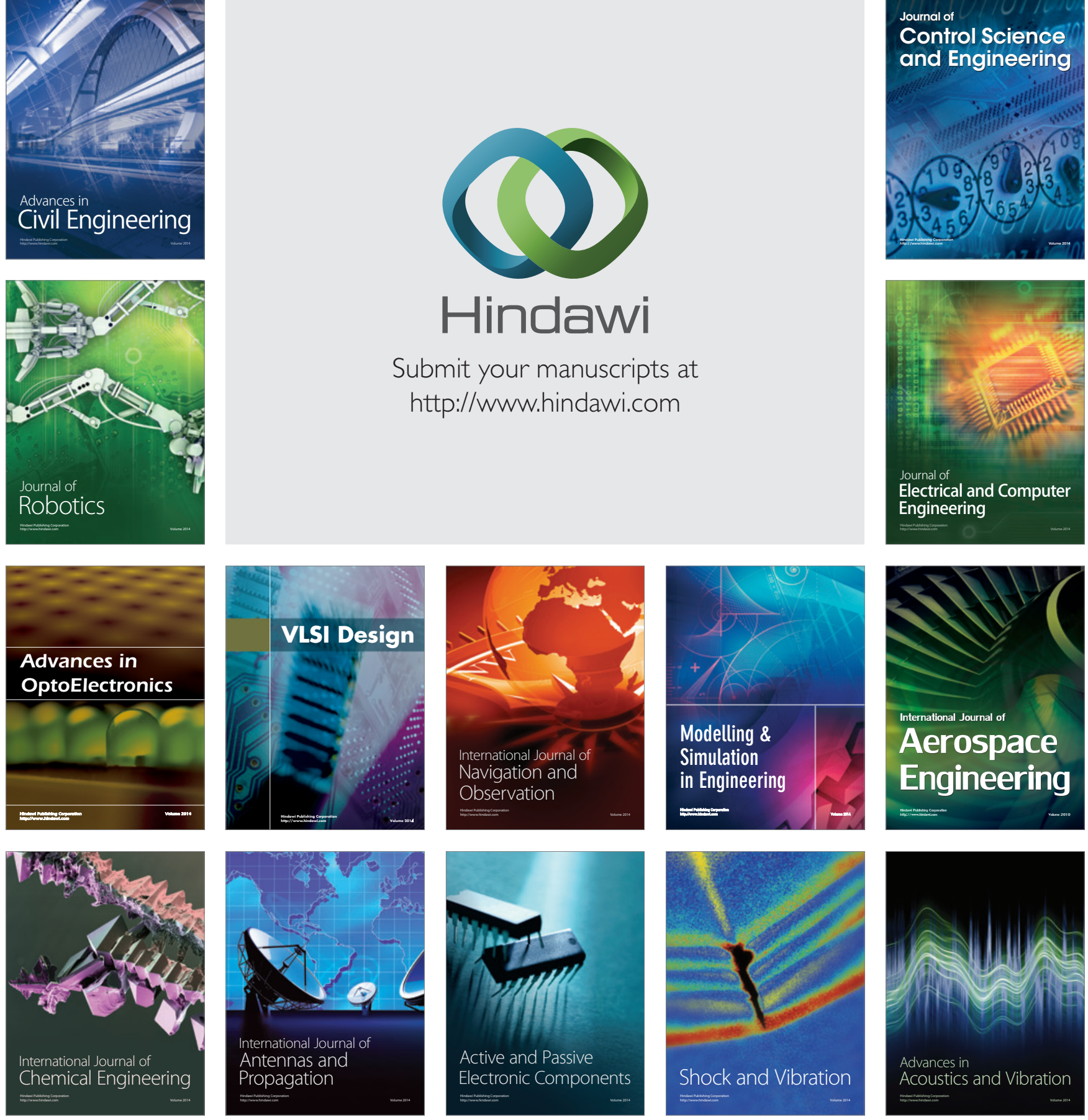